\title{
Una reflexión teórica sobre estándares de áreas verdes empleados en la planeación urbana
}

\section{Green space standards in urban planning: a theoretical reflection}

\author{
RAMiro Flores-Xolocotzi ${ }^{*}$
}

\begin{abstract}
This paper is a theoretical reflection of the use of urban green surface standards used in the praxis of different urban planning models. This article shows that these standards are generally recommended in order to provide welfare to urban dwellers without considering that welfare can be defined according to the rationality paradigm in the planning model, nor does it specify the needs to be covered. In conclusion, there are theoretical-practical limitations in the use of these green surface standards in urban planning.
\end{abstract}

Keywords: cities, green spaces, rationality, sustainability, urbanism.

\section{Resumen}

En esta investigación se realizó una reflexión teórica sobre el uso de estándares de superficie verde citadina empleados en la praxis de algunos modelos de planeación urbana; el trabajo pone de manifiesto que dichos estándares se recomiendan de manera generalizada con el objetivo de proporcionar bienestar a los citadinos, sin considerar que éste se puede definir según el paradigma de racionalidad en el modelo de planeación y sin especificar las necesidades a cubrir; se concluye que existen limitaciones teórico-prácticas en el empleo de dichos estándares de superficie verde en la planeación urbana.

Palabras clave: ciudades, espacios verdes, racionalidad, sustentabilidad, urbanismo.

\footnotetext{
* Universidad La Salle, Ciudad de México, correo-e: pinos42@hotmail.com
} 


\section{Introducción}

En la praxis, la planeación urbana actual tiene que considerar la provisión de diversos bienes y servicios para citadinos; uno de los temas recurrentes en la planeación es la provisión de espacios verdes públicos de libre acceso (Briseño et al., 2010; Echenique et al., 2012). Ante esta situación, las agencias gubernamentales encargadas de gestionar y planear la ciudad se han visto en la necesidad de considerar y emplear diversos estándares que les permitan medir el éxito y alcance de sus programas gubernamentales y de su política pública (Fusco et al., 2009; Paskaleva-Shapira, 2009; Webster y Sanderson, 2012; Young, 2012; Vojnovic, 2014).

En el caso específico de la dotación de espacios verdes han propuesto estándares, principalmente de superficie mínima requerida, para proveer beneficios a la población, considerando que las áreas verdes citadinas ${ }^{1}$ proporcionan diversos beneficios (ecológicos, estéticos, de salud física y psicológica, poblacionales, de recreación, entre otros) (Fan et al., 2011; Young, 2012; Kaźmierczak, 2013; Wolch et al., 2014).

Lo anterior ha derivado en toda una gama de investigaciones y propuestas prácticas sobre áreas verdes urbanas (en particular parques) relacionadas con recreación, impacto ambiental de los espacios, propuestas de diseño arquitectónico, propuestas de planeación, uso de estándares de áreas verdes, medidas de adaptación al cambio climático, entre otros temas (Falcón, 2007; Reyes y Figueroa, 2010; Konijnendijk et al., 2013).

No es de extrañar que las áreas verdes sean consideradas como capaces de contribuir a resolver problemas tan diversos como inseguridad pública, contaminación atmosférica, cambio climático, desintegración social, obesidad generalizada en la población y, en general, problemas relacionados con salud pública (Lee y Maheswaran, 2010; Konijnendijk et al., 2013; Wolch et al., 2014). Es tal el interés sobre el tema, que diversos especialistas recomiendan estándares que las ciudades deben cumplir en cuestión de provisión de superficie verde por habitante (en rangos variables que pueden ir, en algunos casos, desde nueve metros cuadrados por habitante o más) (Sorensen et al., 1998; Gámez, 2005; Wang, 2009; Singh et al., 2010).

Derivado de la importancia de los espacios verdes y del empleo de indicadores de superficie verde en la planeación urbana, este trabajo tiene los siguientes objetivos: identificar y describir la provisión de espacios verdes en modelos históricos y actuales de planeación urbana; describir y

\footnotetext{
${ }^{1}$ En esta investigación se consideran sinónimos los términos áreas verdes y espacios verdes; ambos se refieren sólo a espacios públicos citadinos de libre acceso con diferentes tipos y densidades de vegetación, cuya finalidad es proveer servicios recreativos y servicios ambientales como provisión de oxígeno.
} 
reflexionar teóricamente sobre el uso de los estándares de superficie de espacios verdes en algunos modelos de planeación urbana; considerando lo anterior, describir las limitaciones de dichos estándares en la planeación de áreas verdes y, por lo tanto, en la práctica de la planeación, y finalmente proporcionar recomendaciones que guíen la provisión de espacios verdes hacia otro paradigma de planeación.

\section{Enfoques teóricos de la planeación urbana}

Existen diferentes escuelas de pensamiento sobre planeación urbana, las cuales se sustentan en principios y conceptos económicos, sociales e incluso ambientales. Estos aspectos teóricos determinan la forma en que se entienden y atienden diferentes objetivos encaminados a proveer bienes y servicios para los habitantes de las ciudades, tales como áreas verdes y espacios recreativos.

Para describir y reflexionar sobre la relación entre los modelos de planeación urbana y la provisión de áreas verdes citadinas se integra una breve reseńa histórica (antecedentes) que permite comprender los enfoques de planeación que aquí se discuten; posteriormente, se expondrán los apartados que constituyen este primer punto en el siguiente orden: modelos utópicos, modelos basados en la racionalidad instrumental (comprenden modelos de racionalismo puro y modelos de planeación de tipo neoclásico) y modelos de sustentabilidad urbana.

\subsection{Antecedentes históricos de la planeación urbana}

Previo a abordar los enfoques utópicos es necesario describir brevemente el antecedente histórico descrito por Mumford (2014a) y por Weber (2014) en cuanto a una ciudad y su planeación en tiempos históricos que antecedieron a los modelos propuestos por Ebenezer Howard (Hardy, 2005) y Le Corbusier (Gehl y Svarre, 2013). No es objetivo de este trabajo hacer una detallada revisión histórica de las ciudades, considerando que podrían ser, de acuerdo a Lewis Mumford, cinco mil años de historia urbana, para ello se remite al lector a los trabajos de Mumford (2014a); Weber (2014) y Ponting (1992); sin embargo, es de interés señalar al menos tres etapas de evolución urbana en dicho proceso histórico que son de interés para este trabajo, se consideran las aportaciones de Mumford (2014a) y Weber (2014), estas etapas se describen a continuación. 


\subsubsection{Primera etapa}

La evolución del caserío o aldea hacia una ciudad, esto es, la transición de un territorio de cría y alimentación a una aldea agrícola permanente con una arquitectura y trazado general que comprende calles, espacios y casas ordenadas más por sentido común en un territorio que, posteriormente, devino en una ciudad. La evolución a ciudad comprende, según Mumford (2014a), no sólo un cambio de tamaño y escala sino también un cambio de dirección y propósito expresado en un nuevo tipo de organización caracterizado por un control central (un traspaso de autoridad a la ciudad) que lleva al aldeano a perder su gobierno propio a cambio de una prosperidad y seguridad de la que no gozaba; es en estas primeras ciudades en donde coexisten lentas y complejas interacciones que permiten la emergencia de los primeros esquemas de planeación urbana basados ya no en el sentido común sino en la experimentación y la selección.

\subsubsection{Segunda etapa}

El tránsito del periodo renacentista al barroco, y posteriormente a la emergencia del neoclásico (en el barroco tardío) a mediados del siglo XVIII; estas transiciones implicaron no sólo un cambio de apreciación artística, sino también transformaciones políticas y económicas que influyeron en la planeación urbana (Fernández, 2000). En esta transición se emplearon aspectos matemáticos y abstractos que de acuerdo a Mumford (2014a) se expresaron en rigurosos trazados de calles y planos urbanos, donde los diseños de paisaje eran ordenados geométricamente, estos aspectos coexistieron entre los siglos XVI y XIX.

\subsubsection{Tercera etapa}

El impacto de la Revolución Industrial en las ciudades occidentales, que se manifestó particularmente a mediados del siglo XIX. De acuerdo a Ponting (1992), esta Revolución Industrial creó dentro de las ciudades zonas de contaminación concentrada y de degradación ambiental por el humo y los gases tóxicos emanados de las chimeneas de las fábricas, materiales de desecho en suelos, corrientes de agua contaminadas por residuos industriales y destrucción de la vegetación de las zonas circundantes. ${ }^{2}$

${ }^{2}$ De acuerdo a Mumford (2014a), el crecimiento y desorden en las ciudades del siglo XIX se puede comprender a la luz del utilitarismo basado en el principio del libre mercado para obtener el máximo bien público (laissez faire), donde cada individuo se interesa sólo en lo suyo (egoísmo), este modelo económico que remite a Adam Smith es analizado extensamente por Sen (1977), Basu (2013) y San Emeterio (2014), en mi opinión estos principios de libre mercado están presentes en mayor o menor grado en diversos modelos de planeación urbana que se describen en este trabajo. 
Si bien Mumford (2014a) señala que esta Revolución se manifestó positivamente a través de la incorporación de servicios perfeccionados tales como cañerías de hierro y sistemas de cloacas, estos servicios se pusieron sólo al alcance de los grupos económicos medios y superiores en ciudades europeas y estadounidenses; sin embargo, es en los barrios de la clase obrera donde la misma Revolución se manifestó a través del hacinamiento de personas en viviendas y sótanos húmedos, espacios estrechos entre las casas, poca aireación y luz solar en los espacios públicos, calles sombrías y llenas de basura, situaciones que han sido descritas por Engels (2010), Arias (2012) y Layuno (2013).

\subsection{Modelos utópicos}

Bajo el escenario descrito para las ciudades industriales de mediados del siglo XIX, surge una corriente higienista representada por Florence Nightingale (Young et al., 2011), Louis Pasteur (Caponi, 2002), el Barón Haussmann, entre otros, quienes en palabras de Mumford (2014a) hicieron un esfuerzo por proporcionar soluciones a los problemas a través de la limpieza, el uso de agua corriente y la propuesta de espacios abiertos que permitieran la aireación y la entrada de luz.

En este momento histórico, se puede hablar de los denominados modelos utópicos de la planeación, estos enfoques están representados por autores como Ebenezer Howard y su propuesta Garden City y por las aportaciones de Charles Édouard Jeanneret-Gris Le Corbusier. Igualmente se pueden incluir en estos enfoques las propuestas de planeación desarrolladas en la segunda mitad del siglo XIX por parte de George Eugene Haussmann (Barón Haussmann), en Francia, y las propuestas de planeación y diseńo de parques de Frederick Law Olmsted y Calvert Vaux (creadores del famoso Central Park de la ciudad de Nueva York) en Estados Unidos de América (Garvin, 2010; Mumford, 2014a).

Rodríguez (2011) considera que propuestas como las de Howard y Le Corbusier constituyen un enfoque utópico de la planeación cuyas propuestas tienden a carecer de fundamentos científicos. No obstante, las aportaciones de autores como Ebenezer Howard, Le Corbusier, Haussman y Olmsted tienen aún influencia en propuestas actuales de planeación urbana, incluyendo la provisión de áreas verdes (Gehl y Svarre, 2013; Mumford, 2014a). Las propuestas de Olmsted comprendieron la planeación y diseño de parques urbanos y vías verdes en diferentes ciudades de Estados Unidos (Harnik, 2010; Olmsted, 2015); de acuerdo a Garvin (2010) y Fan et al. (2011) el enfoque de Olmsted se basaba en considerar que los parques proporcionan un efecto relajante al entrar las personas en contacto con paisajes naturales y en facilitar el acceso a 
oportunidades recreativas; proporcionando un efecto educativo y civilizatorio (Olmsted, 2015).

Hay que señalar que el diseño de Central Park, propuesto por Frederick Law Olmsted y Calvert Vaux, estuvo influido, según Garvin (2010) por el diseño de jardines franceses e ingleses del siglo XVIII, estos últimos fueron elaborados por diseñadores de jardines y paisajes como Lancelot Brown (Medina, 2010); sin embargo, la diferencia con dichos jardines es que el parque estadounidense estaba destinado a ser de uso público (Garvin, 2010). Mientras tanto, en ciudades europeas como París, de acuerdo a Garvin (2010), las propuestas de planeación del Barón Haussman que transformaron la ciudad parisina del siglo XIX, demostraron cómo emplear el espacio público abierto como una característica central del desarrollo urbano. El enfoque del Barón Haussmann comprendía cuatro elementos: a) se transformaron dos enormes propiedades de la Corona francesa en parques regionales (hay que recordar que esta transformación urbana ocurrió en el gobierno del emperador Napoleón III); b) establecimiento de tres parques distritales; c) se crearon 24 bloques o manzanas que constituyeron parques vecinales $\mathrm{y} d$ ) se conectaron una red de bulevares arbolados con los parques señalados.

Con respecto a la propuesta de Ebenezer Howard: el Movimiento Garden City, descrito inicialmente en el libro Tomorrow: a peaceful path to real reform, publicado en el ańo 1898 en Inglaterra, sugiere -ante los problemas derivados de la Revolución Industrial en las ciudades inglesasun modelo de ciudad de 32,000 habitantes con una densidad aproximada de 10 a 12 personas por hectárea (Hardy, 2005; Mumford, 2014a). Este modelo de ciudad policéntrica propuso un modelo de ciudad autónoma en la mayoría de servicios y separada de las áreas industriales a través de un cinturón de tierra agrícola de 2,023 hectáreas, lo anterior evitaría la dispersión de la ciudad hacia el campo.

De esta forma, las soluciones de Howard iban dirigidas a contener la descentralización de la población de centros urbanos a la periferia (Steuer, 2000; Hardy, 2005; Vreeker, 2009; Garvin, 2013). El movimiento Garden City antecede a la propuesta estadounidense del movimiento City Beautiful desarrollado por Daniel Burnham, Frederick Law Olmsted Jr. entre otros; este movimiento estuvo dirigido a enaltecer el espacio público de las ciudades estadounidenses en los primeros ańos del siglo XX, teniendo como uno de sus objetivos mejorar el ambiente a través de elementos como los parques (Wilson, 1994; Harnik, 2010).

Esto, a su vez, coincide con las primeras recomendaciones internacionales sobre dotación de áreas verdes citadinas, por ejemplo, en el Segundo congreso internacional de higiene y problemas de urbanismo de París, se señaló como recomendable que $15 \%$ de la superficie de todas las ciudades 
se destinara a parques y jardines y una reserva forestal en sus alrededores con extensión no menor a 10 km (Salvador, 2003); este movimiento tenía entre sus objetivos proporcionar mejores condiciones de habitabilidad que resultaran en mejores rendimientos laborales a través de un control sanitario en viviendas y barrios populares y que incluyeran diversas prácticas sanitarias como la desinfección de edificios públicos (Caponi, 2002; Salvador, 2003).

Posteriormente, en la tercera década del siglo XX, las propuestas de Le Corbusier constituyeron soluciones relacionadas con el incremento de la densidad de las poblaciones urbanas por superficie, estas soluciones se incorporaron en el documento denominado Carta de Atenas, texto que sigue teniendo influencia en el urbanismo del siglo XXI (Vreeker, 2009). Sobre la Carta de Atenas del año 1933 (Instituto Nacional de Cultura, 2007), obtenida a través del Congreso Mundial de Arquitectura, este documento fundamentó una propuesta mundial de planeación urbana basada en principios funcionalistas que promovían la separación de funciones (vivienda, trabajo y recreación). Además, hay que destacar que la Carta de Atenas, en palabras de De Mattos (2004: 12), se convirtió en: "una suerte de texto ritual para la articulación de una poderosa corriente urbanística que ganó adeptos en el mundo entero”.

\subsection{Modelos basados en racionalidad instrumental}

En mi opinión, la Carta de Atenas introdujo a la planeación urbana un enfoque de racionalidad sustantiva o instrumental, este modelo de planeación ha sido denominado como racionalista puro de tipo cientificista, normativo, centralizado (de tipo keynesiano) y unificado para la toma de decisiones, el cual concibe la existencia de un interés urbano público que se puede optimizar para la obtención de un óptimo social (De Mattos, 2004; Rodríguez, 2011; Gutiérrez, 2009; Gutiérrez, 2013). Para entender el enfoque de esta forma de planeación se describe lo que se entiende por racionalidad sustantiva o instrumental, sin embargo, es necesario aclarar que el objetivo de esta descripción no va dirigido a invalidar tal principio de racionalidad ni a la acción racional y los instrumentos que se derivan de este enfoque económico, que se describen posteriormente, sino exponer sus limitaciones en la planeación urbana, especialmente cuando se emplean únicamente estos enfoques económicos particulares para justificar la toma de decisiones en la planeación.

Al considerar lo anterior, la racionalidad sustantiva, como enfoque teórico que explica las motivaciones humanas, es denominada así por Herbert Simon (Simon, 1985; Camagni, 1999), esta denominación coincide con lo que autores como Boudon (2003; 2004), Elster (2003) 
y Crespo (2009) nombran como racionalidad instrumental; bajo este enfoque en la relación medios-fines se concibe a estos últimos como dados y el modelo a seguir -según Coleman (2011) - está definido por actores racionales que maximizan su utilidad a través del intercambio de bienes. Esta relación se puede entender a partir de la teoría de la acción racional ${ }^{3}$ bajo la cual, el homo aeconomicus ${ }^{4}$ optimiza su bienestar, esto es: lo que importa es encontrar los mejores medios para llegar a un fin determinado (Elster, 2003; Cante, 2010).

En consecuencia, este enfoque de optimización del bienestar es la base teórica de la selección de alternativas a través de instrumentos económicos que se emplean en la toma de decisiones de la planeación urbana y de la administración pública, es el caso particular de herramientas como el análisis costo-beneficio. Esta herramienta se puede emplear desde una perspectiva de rentabilidad financiera para empresas privadas; en el caso de su aplicación en el área de la administración pública adquiere otro matiz, ya que el objetivo no es ganar dinero sino incorporar el bienestar, en términos monetarios, de las colectividades, para tomar decisiones de política pública en cuanto a la viabilidad económica y social de proyectos, tal como lo señalan Azqueta (2007), Freeman et al. (2014) y Field y Field (2016). Este análisis costo-beneficio será retomado posteriormente, cuando se describa el paradigma de la sustentabilidad en la planeación urbana.

Aunque el modelo de planeación contaba con un enfoque cientificista que le dotaba de un marco teórico sólido y herramientas para la toma de decisiones centralizadas, como las descritas en el párrafo anterior, hacia los años setenta del siglo XX, el modelo entró en crisis. Esto fue debido, particularmente, al agotamiento de la norma de consumo fordista (consistente en la participación de los ingresos de los trabajadores en el consumo, lo cual conectaba la oferta y demanda de productos) y la crisis del Estado del Bienestar que introducía diversas prestaciones y apoyos, cuyo objetivo era mejorar la vida del trabajador (De Mattos, 2004; Rodríguez, 2011; Gutiérrez y González, 2010). Paralelo a esta situación,

${ }^{3}$ La teoría de acción racional o de elección racional, de acuerdo a Boudon (2003), tiene los siguientes postulados: 1 . cualquier fenómeno social es el efecto de decisiones individuales, acciones, actitudes, etc., (individualismo); 2. en principio al menos, una acción puede ser entendida (comprensión); 3. toda acción tiene una razón (racionalidad); 4. las razones derivan de las consecuencias de las acciones (consecuencialismo, instrumentalismo); 5. los actores se preocupan principalmente de las consecuencias que tienen para sí mismos sus propias acciones (egoísmo); 6. los actores son capaces de distinguir los costos y beneficios de líneas alternativas de acción, la acción con balance más favorable (maximización al preferir una alternativa a otra y su optimización). De acuerdo a Boudon (2004) la teoría de acción racional es un subconjunto basado en la racionalidad instrumental. Además, González (2011) sugiere como condición del modelo clásico de elección racional las preferencias transitivas de los agentes económicos.

${ }^{4}$ De acuerdo a Pedrajas (2006: 13), el homo aeconomicus es "aquel ser egoísta que actúa movido únicamente por su propio interés. Es un agente calculador y maximizador de su propio beneficio que por la lógica de la mano invisible consigue el beneficio social”. 
emerge un nuevo discurso económico de tipo neoclásico, del cual, una de sus características es favorecer el libre mercado moderando la intervención gubernamental, idea opuesta a la visión keynesiana del Estado de Bienestar.

Lo anterior coincidió con un replanteamiento del estudio económico del espacio urbano (economía espacial) que cobra fuerza a través de las aportaciones de Paul Krugman, las cuales permiten analizar la concentración espacial de las actividades económicas a través de modelos de competencia monopolística, pero que mantienen el supuesto de optimización máxima de la utilidad sujeta a una restricción presupuestal (Camagni, 1999; Fujita et al., 2001).

De esta forma, el discurso económico neoclásico y la competitividad espacial contribuyeron a desarrollar y fortalecer nuevas propuestas de planeación urbana, por ejemplo, hacia los años ochenta, surgen enfoques como el Modelo de Planeación Incrementalista propuesto por Charles Lindblom y descrito por Rodríguez (2011) que, sin renunciar a los principios de la racionalidad instrumental, considera que las personas son seres racionales capaces de tomar las mejores decisiones; su acercamiento a la planeación es a través de una metodología sin coordinación central, basada en aproximaciones sucesivas, lo cual lleva los principios de la economía de libre mercado al ámbito de la planeación urbana.

Por ello, en mi opinión, el principio de libre mercado en el modelo de planeación de Lindblom o en otros modelos de corte similar, permite que el enfoque global de racionalidad sustantiva o instrumental cobre mayor fuerza en las propuestas de planeación, manteniendo intacto el principio del homo aeconomicus basado en la acción racional. Al respecto, De Mattos (2004) señala que el enfoque instrumental de planeación y de libre mercado se extendió en los años noventa del siglo XX en diversas ciudades del mundo occidental que buscaban la competitividad, constituyendo lo que se denomina urbanismo empresarial.

Por lo mismo, se hace necesario mencionar algunos de los efectos de esta racionalidad instrumental y de libre mercado en las ciudades y sus espacios públicos que incluyen a las áreas verdes; al respecto, se menciona que la planeación urbana basada exclusivamente en un enfoque racionalista instrumental y de libre mercado sienta las bases de lo que autores como Jirón y Mansilla (2014) han denominado urbanismo fragmentador (de tipo físico/espacial). Éste consiste en prácticas de intervención que fragmentan la vida cotidiana de los citadinos, lo anterior puede ser causado por uno o más de los siguientes aspectos: políticas que fomentan el uso del automóvil (Mumford, 2014b); formas de intervención urbana diferenciada según zonas de la ciudad que acentúan la segregación social (imponiendo barreras al desplazamiento) y una expansión inmobiliaria y 
de mega infraestructuras que inciden en la fragmentación (Jirón y Mansilla, 2014) y que, de acuerdo a Salvador (2003), ha conducido con el tiempo a la reducción de espacios verdes citadinos, debido a su combinación con una perspectiva funcionalista (separación de funciones: vivienda, recreo, trabajo y otros) y a la especulación que produjo en la realidad la zonificación de ciudades que se plantearon inicialmente en la Carta de Atenas durante la época de Le Corbusier.

Bajo estos supuestos, en mi opinión, la provisión de servicios públicos como los espacios verdes, son vistos bajo estos enfoques de planeación como parte de un modelo económico de eficiencia, en el cual la cantidad y ubicación de espacios verdes debe ser optimizada para incrementar el bienestar de los citadinos, considerando criterios de competitividad territorial que llevan además al rescate y embellecimiento de los espacios urbanos en pos de la competitividad, tal como lo señala De Mattos (2004).

Los efectos descritos en el párrafo anterior pudieran explicarse debido a la concepción de un interés público citadino que debe ser optimizado, lo cual puede llevar de manera obligada a estimar dicho interés y correr el riesgo de reducir el proceso de toma de decisiones citadinas exclusivamente a métodos basados en la racionalidad sustantiva e instrumental, tales como el análisis beneficio-costo. Estos métodos están acotados por las mismas limitantes de un modelo de conducta basado en la acción racional que concibe al individuo con medios y fines dados, olvidando las propias limitantes de un modelo de racionalidad instrumental y de libre mercado. $\mathrm{Al}$ respecto, en este trabajo se coincide con lo externado por Elinor Ostrom, Herbert Simon y Amartya Sen, en el sentido de que no se puede universalizar un modelo específico de conducta humana que concibe al ser humano como un homo aeconomicus que actúa según los supuestos de la acción racional en un marco de racionalidad instrumental (Sen, 1977; Simon, 1985; Ostrom, 2010; Ostrom, 2015).

Los argumentos que los autores mencionados obtienen a partir de experimentos, trabajo de campo y análisis de resultados, les permiten proponer enfoques teóricos complementarios a la teoría de acción racional (de corte instrumental) bajo un concepto más amplio de racionalidad -tal como lo señalan Crespo (2009) y Calvo (2012) - que considera capacidades cognitivas limitadas en los individuos (Simon, 1985) y toma en cuenta procesos cooperativos a través de acuerdos o arreglos institucionales en individuos con racionalidades limitadas (Ostrom, 2010; 2015), o bien, considera una racionalidad económica que integra como parte de su estructura los sentimientos de los agentes económicos hacia el bienestar de los otros (Sen, 1977).

Sobre esto, autores como Cante (2010), Salas (2013), Basu (2013) y Williamson (2013) hacen una crítica del enfoque de la acción racional y 
del homo aeconomicus, y la dirigen a proponer una nueva forma de concebir los fines, consideran la posibilidad de redefinir los fines en el sentido de North $(1993 ; 2006)$ ya que existe una amplia gama de actividades o acciones humanas no maximizadoras de riqueza como las relaciones afectivas, sociales o aspectos culturales, por lo que las motivaciones de los agentes económicos son más complejas. Es posible también contemplar una amplitud de fines que guían nuestras acciones personales y colectivas como costumbres, prestigio, razones emotivas, afectivas, el azar, capricho, entre otros; de esta forma, un modelo de racionalidad más amplio, como lo sugiere Crespo (2009), permitiría un distanciamiento de la tradicional visión puramente económica neoclásica, acercándose así a la perspectiva del nuevo institucionalismo.

En mi opinión, las implicaciones prácticas de lo anterior, desde una perspectiva económica, permitirían concebir de distinta forma las finalidades públicas y colectivas dentro de la planeación urbana y sus programas de dotación de servicios públicos, por lo que es necesario abrir a la crítica y al debate académico, la concepción teórica que relaciona a la economía y a la planeación urbana como dominios académicos puramente técnicos y de medios eficaces basados en la acción racional y su racionalidad instrumental.

Bajo este escenario, es posible identificar algunos eventos que prepararon el camino hacia enfoques actuales de planeación urbana a nivel mundial. El primer evento fue el acuerdo y publicación de la Carta de Machu Picchu en 1977, cuya finalidad fue modernizar la Carta de Atenas, más no negarla (Instituto Nacional de Cultura, 2007). A diferencia de la Carta de Atenas, en este nuevo documento se llama a tomar conciencia en torno a que la planeación urbana no consiste en sectorizar a través de las cuatro funciones básicas descritas por la Carta de Atenas (habitar, trabajar, recrear y circular), sino en desarrollar una integración polifuncional y considerar el crecimiento demográfico acelerado que no se consideró en la primera carta (Instituto Nacional de Cultura, 2007).

El segundo suceso fue de alcance político mundial, ya que en 1988, la Organización de las Naciones Unidas (ONU) publicó el Informe Brundtland, en este documento se propuso el concepto de desarrollo sustentable como política pública a incorporar en los procesos de planeación urbana, entendiendo dicho desarrollo como aquél que permitía satisfacer las necesidades del presente sin comprometer la capacidad de satisfacción de necesidades de las generaciones futuras (Mebratu, 1998; WCED, 2009).

De acuerdo a Lozano (2008) y a Rocuts et al. (2009), el desarrollo sustentable comprende una compleja interrelación de aspectos económicos, ambientales y sociales que en el caso de la visión de Brundtland se 
ciñen a un ambientalismo moderado, tal como se describirá en el siguiente apartado. Por otra parte, en 2003, el Consejo Europeo de Urbanistas desarrolló y publicó la Nueva Carta de Atenas (Instituto Nacional de Cultura, 2007), esta carta consideró la diversidad cultural y lingüística de las urbes europeas actuales y sus problemas ambientales y sociales; señala que la planeación del espacio es vital para proporcionar un desarrollo sustentable e introdujo el principio de proximidad para resaltar la necesidad de que los citadinos vivan conectados con elementos bien conservados del patrimonio natural, el cual comprende el espacio público.

Consecuentemente, de acuerdo a Fernández (2006), los paradigmas neoclásicos de la planeación existentes en la última década del siglo XX, y cuyos objetivos principales eran incrementar la competitividad de las ciudades en un ámbito económico y mejorar las condiciones de habitabilidad en los ámbitos espacial y social, incorporan en su construcción teórica y práctica, el concepto de desarrollo sustentable, tal como se describe en el siguiente apartado.

\subsection{Modelos de planeación urbana sustentable}

De acuerdo con Camagni (1999; 2005), el desarrollo sustentable en ciudades se puede entender a través de un par de dicotomías: a) el enfoque teórico de racionalidad y b) el enfoque teórico de lo que se entiende como sustentabilidad. La primera dicotomía que comprende el enfoque de racionalidad se haya representado en este trabajo al describir la propuesta de racionalidad instrumental y de la acción racional, junto a propuestas teóricas complementarias que surgen a partir de un enfoque amplio de lo que es la racionalidad. La segunda dicotomía comprende dos formas de definir la sustentabilidad: débil contra fuerte (Camagni, 1999; Pierri, 2005).

$\mathrm{Al}$ considerar lo anterior, este trabajo coincide con Barton (2006) al señalar que existen dos formas de entender la planeación urbana sustentable: a) según la sustentabilidad débil y b) según la sustentabilidad fuerte, las cuales deben entenderse en función de grado de muy débil a muy fuerte según el nivel de sustitución permitida entre los factores de producción (capital manufacturado frente a capital natural). Sobre esta dicotomía, se señala que no es objetivo de esta investigación hacer una revisión integral y completa de todos los principios teóricos que comprenden la sustentabilidad débil y de la sustentabilidad fuerte, lo cual va más allá de los alcances de este escrito, pero sí es de interés para este trabajo destacar las diferencias en las propuestas de racionalidad que subyacen bajo los enfoques de la sustentabilidad débil (economía ambiental) y de la sustentabilidad fuerte (economía ecológica), las cuales se describen a continuación. 
De acuerdo con Pierri (2005) la sustentabilidad débil comprende los supuestos teóricos de la economía ambiental neoclásica con cierto matiz keynesiano, por lo mismo, es opinión del autor de este trabajo que la sustentabilidad débil, representada por la economía ambiental, descansa sobre un enfoque de racionalidad instrumental y de la acción racional, por lo mismo constituiría el enfoque de planeación urbana neoclásico descrito por Barton (2006) y, que de forma coincidente con él, no es favorecido en las reflexiones teóricas que se hacen aquí debido a aspectos que se mencionan a continuación.

El enfoque de planeación urbana que se deriva de la sustentabilidad débil hace énfasis en tiempos de planeación a corto plazo, que pueden coincidir con los tiempos de la gestión gubernamental (Barton, 2006), además, este enfoque tiene una orientación basada principalmente en las actividades económicas citadinas y de iniciativas empresariales innovadoras, lo que deja como objetivos posteriores aspectos de habitabilidad relacionados con el desarrollo de ambientes favorables para el citadino y la comunidad.

Para lograrlo se hace uso de instrumentos económicos proporcionados por la economía ambiental; entre estos instrumentos se incluyen análisis costo-beneficio que integran valores ambientales. La economía ambiental tiene como uno de sus objetivos estimar monetariamente los beneficios y costos que se derivan de las acciones humanas en relación con el ambiente y que, la mayoría de las veces, carecen de precio de mercado; tales estimaciones pueden ser incorporadas al análisis costo-beneficio anteriormente mencionado (Freeman et al., 2014; Field y Field, 2016). Actualmente, para conocer dicho bienestar económico se recurre a métodos de valoración de bienes y servicios ambientales, es el caso del Método de Valoración Contingente, el cual es descrito a detalle por Venkatachalam (2004); Whitehead y Blomquist (2009); Nunes y Nijkamp (2011); Zhang y Zhou (2012)

Por otra parte, este trabajo coincide con Pierri (2005), respecto a que la posición teórica de la economía ambiental representa un ambientalismo moderado bajo el cual se acepta la sustituibilidad entre tipos de capital, lo que permitiría un crecimiento económico sujeto a una restricción de conservación de recursos que evite la degradación ambiental; igualmente, el Informe Brundtland puede considerarse como una serie de políticas recomendadas bajo la base del mismo ambientalismo moderado (Pierri,

\footnotetext{
${ }^{5}$ Este método, fundamentado por la teoría microeconómica, permite conocer los valores monetarios expresados por los beneficiarios de bienes públicos, tales valores resultan del ordenamiento de las preferencias de los consumidores siguiendo el enfoque de la ya citada teoría de la acción racional (Azqueta, 2007; Freeman et al. (2014). Además, la valoración contingente es uno de los métodos más empleados para conocer el valor monetario de los servicios que proporcionan los espacios verdes urbanos (Jim y Chen, 2006; Frutos de y Esteban, 2009).
} 
2005), que concibe el mismo modelo de crecimiento, sustitución, conservación y no degradación de la economía ambiental, y que si bien no invalida perspectivas como la de la economía ecológica-que se describe a continuación- sí las puede ceñir a sus propuestas de política urbana.

Sobre sustentabilidad fuerte se sigue a Martínez y Roca (2001), Pierri (2005), Hernández (2008) y Daly (2013); este enfoque se inscribe en la propuesta teórica de la economía ecológica, la cual hace una crítica al modelo económico neoclásico empleando principios de la ecología y termodinámica, por lo mismo concibe un crecimiento económico limitado a la biósfera y una no sustituibilidad entre capital manufacturado y natural en las funciones de producción; al considerar estos principios, una expresión práctica de este enfoque es el indicador denominado como huella ecológica. ${ }^{6}$

Barton (2006) extiende la descripción de sustentabilidad fuerte en planeación urbana al mencionar ciertas cualidades, a las cuales este trabajo de planeación de áreas verdes muestra afinidad, tales características son horizontes de planeación a largo plazo de hasta 100 años, los cuales van más allá de los tiempos administrativos de gestión pública de los gobiernos locales; además de tener como uno de sus objetivos principales una localidad urbana con una visión de compasión; buena gestión de recursos naturales y construcción de vínculos con otras localidades.

Por su parte, Martínez y Roca (2001) señalan que la economía ecológica por su carácter multidisciplinario incorpora en su método marcos multicriterio para la toma de decisiones, por lo que se abre a incorporar diversos criterios económicos, ambientales y hasta sociales en la toma de decisiones; en mi opinión, esta característica metodológica de la economía ecológica permitiría abordar la planeación de áreas verdes y su toma de decisiones sin ceñirse sólo a instrumentos como el costo-beneficio o bien sólo a estándares cuantitativos que están ya limitados en cuanto a lo que pueden expresar respecto al bienestar de las personas, como se describió con anterioridad.

A las cualidades enunciadas sobre la economía ecológica se añaden críticas o señalamientos sobre una ausencia de modelos de comportamiento o motivaciones humanas que permiten explicar las decisiones ambientales

${ }^{6}$ De acuerdo a Wackernagel y Rees (2001: 26): "el análisis de la huella ecológica es una herramienta contable que nos permite estimar los requerimientos en términos de consumo de recursos y asimilación de desechos de una determinada población o economía, expresados en áreas de tierra productiva". Con respecto a huella ecológica (HE), de acuerdo a Meadows et al. (2006: 450-451), Wackernagel define su HE como la superficie de terreno necesaria para sostener el nivel de vida actual. Su HE se mide en hectáreas (en promedio mundial). Suma la extensión de tierras de cultivo, pastizales, caladeros de pesca y terrenos edificados que se precisan para mantener a una población dada (país, región, mundo) con un estilo de vida dado. Suma la extensión forestal necesaria que se precisaría para absorber el dióxido de carbono emitido por la energía fósil utilizada por la población. 
que toman los individuos (Lehtonen, 2004; Venkatachalam, 2007; Kallis y Norgaard, 2010). Considerando lo anterior, en este trabajo se coincide con lo señalado por Venkatachalam (2007), respecto a que la economía ecológica necesita incorporar un modelo de racionalidad que vaya más allá de la acción racional que fundamenta al modelo económico neoclásico. Para ello, autores como Lehtonen (2004) han descrito puentes que unen a la economía ecológica con el neoinstitucionalismo económico a través del interés mutuo en el estudio y análisis de los arreglos institucionales de las comunidades humanas sobre el ambiente y sus recursos naturales, es el caso de la perspectiva desarrollada por Elinor Ostrom, a quien autores como Wall (2014) relacionan con la perspectiva de la economía ecológica; por otra parte autores como Venkatachalam (2007) y Kallis y Norgaard (2010) argumentan que el modelo de racionalidad limitada de Simon puede proveer fundamentos conductuales a la economía ecológica.

De la misma manera, este trabajo coincide con Venkatachalam (2007) en cuanto a la necesidad de considerar el impacto que puede tener el diseño de una política ambiental elaborada en términos de un modelo de racionalidad neoclásico sobre individuos que, en su relación con el ambiente o los recursos naturales, siguen otro modelo de racionalidad. Este trabajo hace extensiva esta observación al campo de la planeación urbana como disciplina en donde se proponen políticas de provisión de bienes públicos, por lo que es necesario abrir estos aspectos al debate teórico y a la investigación empírica en los estudios de planeación urbana y su praxis en las ciudades. Considerando la praxis mencionada, Pierri (2005) sugiere que las propuestas de sustentabilidad pueden operar situándose en un punto intermedio entre economía ambiental y economía ecológica.

Por lo cual, en mi opinión, las cualidades de la economía ecológica podrían ayudar a construir un nuevo modelo de planeación urbana que incorpore diversos aspectos de este enfoque de sustentabilidad en propuestas de política ambiental y planeación, sin ceñirse solo a una perspectiva económica de racionalidad instrumental en la toma de decisiones. Lo anterior podría reconceptualizar la provisión de espacios verdes urbanos de libre acceso, lo que permitirá delimitar el uso de estándares de superficie verde por persona e integrar al análisis otros aspectos sociales y ambientales que no se han discutido en este trabajo pero que se dejan para futuras reflexiones, debates e investigaciones, por ejemplo: tipos de recreación, participación ciudadana, inclusión de grupos vulnerables, gestión de áreas verdes, financiamiento, salud pública, recarga de mantos acuíferos, control de inundaciones, conservación de biodiversidad, cambio climático, entre otras (Fan et al., 2011; Webster y Sanderson, 2012; Kaźmierczak, 2013). 


\section{Reflexiones sobre el uso de estándares de áreas verdes urbanas}

La caracterización de los modelos de planeación descritos en el punto anterior, junto con la descripción histórica de su relación con la provisión de espacios verdes, permite establecer en este trabajo que -bajo un enfoque estrictamente racionalista puro- los estándares de planeación de áreas verdes cobran fundamental importancia en aspectos de optimización del bienestar de los habitantes de las ciudades. Por ejemplo, en la década de los cuarenta del siglo XX, Patrick Abercrombie (1943) realizó un ejercicio comparativo de superficie verde por mil habitantes en diferentes condados ingleses y estableció en su propuesta de planeación urbana para la ciudad de Londres, Inglaterra, un estándar que él denominó como cifra razonable a alcanzar (en términos de provisión) de 1.62 hectáreas de superficie verde por mil habitantes.

De acuerdo a Abercrombie (1943), este estándar sería cubierto considerando lo que él denomina espacio verde abierto, que comprende diferentes tipos de espacios: centros deportivos, parques recreativos, vías peatonales verdes, áreas recreativas infantiles, canchas deportivas, campos escolares deportivos, entre otros. No obstante, Abercrombie indica que "no es posible" establecer cifras exactas de superficie necesaria para cada uno de los tipos de espacio verde. Sin embargo, el mismo autor sugiere que para espacios deportivos - canchas, campos escolares y centros deportivos- son necesarias $8094 \mathrm{~m}^{2}$ por cada 1000 habitantes, mientras que para otros espacios -como grandes parques recreativos, vías verdes, áreas de juegos infantiles, pequeños parques- el estándar recomendado fluctuaría de $1348 \mathrm{~m}^{2}$ a $2698 \mathrm{~m}^{2}$ para cubrir la superficie restante.

Finalmente, hay que destacar que en la estrategia de Patrick Abercrombie los espacios verdes tienden a ser un aspecto de importancia en su propuesta de planeación urbana, de tal forma que constituye un sistema integral de espacios intercomunicados dirigido a cubrir servicios (recreativos y de salud) de importancia para los habitantes.

Con este panorama, y ante la imposibilidad de tener cifras exactas de superficies necesarias por tipo de espacio, cabe destacar que la superficie de espacio verde que se considera necesaria para los habitantes pudiera estar determinada en mayor o menor grado por criterios personales del planificador o planificadores. Lo anterior ha permitido que autores diversos propongan tales estándares y que en algunos casos esos estándares se recomienden como mínimos universales. Diversos estándares cuantitativos se han propuesto a la fecha, en mi opinión, desde un enfoque instrumental optimizador tales estándares se puedan emplear para generar esquemas de provisión y evaluación de objetivos de planeación relacionados con espacios verdes. Con fines de ejemplo, se señalan los siguientes estándares 
de superficie verde por persona, que han sido propuestos por diversos autores en Europa y Estados Unidos de América:

a) En Francia en 1973 se recomendó como política pública de áreas verdes urbanas un estándar de superficie verde de $25 \mathrm{~m}^{2} /$ habitante, sin especificar el tipo de servicio ambiental o recreativo que dicho estándar debe cubrir, ni tampoco si son de acceso libre (Bougé, 2009). Sin embargo, Salvador (2003) cita que esos $25 \mathrm{~m}^{2} /$ habitante se refieren a zonas forestales urbanas europeas y tienen una finalidad ecológica. Con fines ecológicos más específicos, Singh et al. (2010) y Wang (2009) reportan un estándar recomendado de áreas verdes de hasta $40 \mathrm{~m}^{2} /$ habitante con fines de provisión de oxígeno y captura de carbono para Alemania y Japón, sin especificar si esas áreas comprenden sólo las de libre acceso o qué tipo de áreas comprenden.

b) Por su parte, en España, Salvador (2003) cita que desde la década de los noventas del siglo XX el estándar de superficie se situaba entre 8 y $12 \mathrm{~m}^{2} /$ habitante, se contemplaba cubrir aspectos culturales, estéticos y de recreación; el estándar tampoco especifica si son espacios de libre acceso o si incluye o no espacios privados, por ejemplo jardines o huertos caseros.

c) En años recientes, la ONU (2015) señala que la Organización Mundial de la Salud (WHO por sus siglas en inglés), recomienda mundialmente un estándar mínimo de $9 \mathrm{~m}^{2}$ de área verde urbana por habitante, la recomendación no especifica qué tipo de servicios cubrirá el estándar, tampoco define si comprende sólo espacios públicos de libre acceso. No obstante, en el año 2010, la WHO señaló que no existe una regla de espacio verde urbano mínimo por habitante (WHO, 2010).

d) Finalmente, Dahl y Molnar (2003) establecen que en ciudades es necesario hablar de sistema de parques (entendido como un conjunto de ellos), lo anterior debido a la imposibilidad de que un parque urbano cubra todas las necesidades recreativas de los usuarios. Sobre dicho sistema de parques, Dahl y Molnar (2003) sugieren un estándar de $40.5 \mathrm{~m}^{2}$ de superficie de parque por habitante.

Sobre el empleo de estos estándares hay que señalar algunos aspectos que se han sugerido en la planeación de los espacios verdes citadinos, por ejemplo, Salvador (2003) y Gámez (2005) sugieren la necesidad de disponer de estándares de áreas verdes basados en tipologías (clasificación o tipos de área verde) que tiendan a ser universales, estables y prácticos, 
tales estándares deberían expresar el bienestar de la comunidad urbana. Consecuentemente, se ha hecho énfasis en que, para cumplir su objetivo, la planeación de áreas verdes citadinas requiere una tipología de espacios verdes que defina dichos diferentes espacios verdes con base en características, funciones y servicios que las áreas verdes prestan, tales como:

a) Superficie del espacio.

b) Funciones ecológicas derivadas de los bienes y servicios ambientales que proporcionan los espacios verdes, no obstante, se tiene que considerar que las ciudades presentan diferentes tipos vegetacionales, ubicaciones geográficas y una amplia diversidad de climas (templados, tropicales, áridos) por lo mismo, el impacto ecológico y ambiental de dichas funciones dependería del tipo de vegetación presente en el espacio verde.

c) Funciones sociales, basadas por ejemplo en los servicios recreativos que puede prestar y el tipo de visitantes que hacen uso del espacio, los cuales pueden ser muy diversos.

Sin embargo, en mi opinión, aunque los aspectos señalados sugieren que los criterios deben expresar las necesidades y bienestar de la población que se derivan de cada tipo de servicio, no se considera que la definición de bienestar -que se quiere proporcionar a los habitantes de las ciudades a través de la planeación-depende del enfoque de racionalidad que posea el modelo de planeación.

Igualmente se recomienda su empleo de manera generalizada, sin especificar si el estándar comprende espacios públicos o privados (es el caso de jardines o huertos caseros), el tamaño de la ciudad y su población, su superficie y su densidad poblacional, por dar sólo algunos ejemplos. También, y de acuerdo con McLoughlin (1969), se tiene que considerar que los estándares de provisión de superficie de espacios verdes por habitante fallan en reflejar las necesidades y la composición social de las comunidades. Consecuentemente, existe la posibilidad de querer homologar y generalizar los gustos, preferencias y necesidades de los citadinos sólo a través de estándares de superficie verde per cápita, sin considerar las diferencias sociales, económicas, geográficas y ambientales de las ciudades y de sus habitantes.

Al respecto, Harnik (2010) considera que la discusión y la planeación de los espacios verdes no se debe limitar a la cantidad de superficie verde por habitante con base en esquemas "aparentemente científicos". Harnik (2010) argumenta que se tienen que tomar en cuenta aspectos como: a) que muchas de las ciudades ya están construidas; b) que las ciudades tienen una amplia diversidad de densidades poblacionales y formas, c) 
que los habitantes tienen diferentes formas de vivir, d) que las densidades poblacionales afectan la forma en que la gente vive su vida, esto es, a mayores densidades se favorecen las interacciones humanas y al mismo tiempo se generan potenciales conflictos derivados del hacinamiento: ruido, privacidad y demanda por instalaciones para la recreación; e) que los espacios están interconectados con otros factores de la planeación: la vivienda, las vías de comunicación, el ancho de las calles, zonas de comercio y tiendas, lenguaje, cultura, zonas escolares, entre otras.

Algunos aspectos sobre el uso de estándares empleados en la planeación de áreas verdes y parques han sido también señalados por Garvin (2013), quien menciona que los estándares han servido en la gestión pública para competir por presupuestos en las oficinas burocráticas y para justificar los departamentos burocráticos dedicados a la gestión y planeación verde, asimismo, ayudan a comparar la demanda por servicios recreativos con niveles de financiamiento políticamente alcanzables.

Harnik (2010) acota el empleo de los estándares de espacio verde por habitante a ser sólo elementos que puedan servir para comparar la provisión de superficie en ciudades con densidades poblacionales similares. De otra forma, la comparación entre ciudades con diferentes densidades poblacionales no tendría caso, por lo mismo se correría el riesgo de interpretar erróneamente las recomendaciones de estándares internacionales, lo cual ha sido descrito por Garvin (2013), quien sugiere que una lectura incorrecta puede provocar que el planificador recomiende incrementar la superficie verde por persona en lugares con baja densidad poblacional y alta cantidad de espacio privado, o bien, sugerir incrementar la superficie verde per cápita incluso por arriba de la superficie total de una ciudad.

\section{Limitaciones en el empleo de estándares en la planeación de áreas verdes}

Tras lo anterior, la propuesta y uso de estándares de superficie verde citadina debería especificar los siguientes aspectos:

a) La necesidad social, ambiental, recreativa, paisajística e incluso de salud pública que se quiere abordar y cubrir a través del estándar de espacios verdes que se sugiere.

b) Especificar si el estándar comprende sólo espacios de libre acceso o bien espacios de acceso restringido por ser privados (jardines o huertos caseros) o de difícil acceso.

c) Limitar sus alcances de aplicación considerando la diversidad, geográfica, ambiental, extensión superficial, tamańo y densidad poblacional de las diversas ciudades en donde su pudiera aplicar. 
d) La diversidad de usos, recreativos que se pueden realizar en los espacios verdes urbanos; sin embargo, al ser los tipos de recreación tan vastos, éstos limitan el desarrollo de un posible estándar universal de mínima superficie verde con fines recreativos por persona en ciudades.

La falta de especificación de los aspectos anteriores, en mi opinión, demuestra que a pesar de las limitaciones teóricas y prácticas que pudiera tener el uso de los estándares de mínima superficie verde por persona en planeación urbana -actualmente diversos estándares de áreas verdes urbanas, especialmente el de la $\mathrm{WHO}$ - éstos se siguen sugiriendo de forma generalizada (Bascuñán et al., 2007).

De esta manera, si tales estándares se emplean con fines de optimización del bienestar, ellos están limitados por la perspectiva o forma en que la misma teoría de la acción racional concibe al bienestar del individuo y las colectividades para las que proveen el servicio público de los espacios verdes, concibiéndose sólo desde una perspectiva económica optimizadora de racionalidad instrumental. No se considera que ese bienestar se puede concebir también desde otros paradigmas que se han desarrollado y se están debatiendo actualmente en economía (Crespo, 2009; Gordillo, 2014).

Este trabajo coincide con la importancia social y ambiental que pueden tener los espacios verdes urbanos. Sin embargo, el uso exclusivo y generalizado de los estándares de superficie verde por persona, en la planeación de áreas verdes, corre el riesgo de restringirse a una toma de decisiones basada sólo en una perspectiva económica optimizadora; se corre el riesgo de desarrollar una política de provisión de espacios verdes basada sólo en una racionalidad instrumental y en la acción racional, mientras que los beneficiarios pueden actuar siguiendo otro modelo de racionalidad tal como lo señala Venkatachalam (2007).

En este punto, la economía ecológica pudiera proveer un marco integral que incorpore una propuesta complementaria de racionalidad, de bienestar y de toma de decisiones. Emplear de manera aislada tales estándares de superficie verde pudiera pasar por alto necesidades específicas de los diversos citadinos que hacen uso de ellos, por ejemplo aquéllas ligadas a relaciones sociales o recreación. Por lo mismo, es necesario abrir al debate científico e impulsar la investigación sobre la posible relación existente entre políticas de provisión de espacios verdes y la racionalidad subyacente en los modelos de planeación urbana actual y discutir la pertinencia de seguir empleando recomendaciones generalizadas de estándares de superficie verde por persona, las cuales presentan limitaciones teóricas y prácticas. 
Es necesario incorporar al debate los paradigmas de sustentabilidad (fuerte y débil) y las propuestas teóricas desarrolladas por Amartya Sen, Herbert Simon y Elinor Ostrom (Crespo, 2009; Pedrajas, 2006; González, 2011; Salas, 2013; Gordillo, 2014), cuyas implicaciones en propuestas de planeación urbana y política pública de bienes como las áreas verdes, tendrán que ser analizadas y debatidas en futuras investigaciones.

\section{Conclusiones}

Junto con las bondades tecnológicas derivadas de la Revolución Industrial, las ciudades occidentales del siglo XIX se caracterizaron por presentar diversos problemas que afectaron particularmente a la clase obrera, lo anterior provocó que el espacio público y las áreas verdes urbanas, incluyendo el diseño de parques, cobraran importancia en las primeras estrategias dirigidas a cambiar la naturaleza sombría de las ciudades del siglo XIX.

Con el inicio del nuevo siglo, los espacios verdes fueron parte fundamental de diversos modelos utópicos, su importancia se ve reflejada en las recomendaciones internacionales sobre superficie de áreas verdes encaminadas a proporcionar un impacto en la salud de los citadinos en la primera década del siglo XX. Lo anterior antecede a las propuestas de Le Corbusier y de la Carta de Atenas en la década de los treintas, que precedieron a los primeros modelos de planeación de racionalismo puro, a través de los cuales se fincó una visión racional instrumental que concebía un interés público unitario que debe ser optimizado para incrementar el bienestar bajo una concepción económica particular de esa racionalidad.

De esta forma, a finales del siglo pasado, los modelos de planeación evolucionaron hacia propuestas actuales con principios de económica neoclásica y posteriormente con los paradigmas de sustentabilidad débil (economía ambiental) o fuerte (economía ecológica). Bajo estas nuevas propuestas se siguen haciendo recomendaciones generalizadas de superficie verde por persona como criterio para toma decisiones. Esos estándares tienen limitaciones teórico prácticas y su uso generalizado podría restringir la provisión de espacios verdes a una concepción particular de bienestar, sin considerar otros criterios de importancia social y ambiental. Por ello, es necesario abrir al debate científico los paradigmas de racionalidad y sustentabilidad en la planeación urbana y discutir la pertinencia de seguir empleando recomendaciones generalizadas de estándares de superficie verde por persona. 


\section{Fuentes consultadas}

Abercrombie, Patrick (1943), "The 1943 Abercrombie Plan for the County of London", Nightingale Garden Company Limited, Londres, Inglaterra, $<\mathrm{http} / / \mathrm{ww} w$. londonlandscape.gre.ac.uk/abercrombie. htm>, 12 de febrero de 2017.

Arias, Walter (2012), "Revisión histórica de la salud ocupacional y la seguridad industrial", Revista Cubana de Saludy Trabajo, 13 (3), Instituto Nacional de Salud de los Trabajadores, La Habana, Cuba, pp. $45-52$.

Azqueta, Diego (2007), Introducción a la Economía Ambiental, McGrawHill, Madrid, España.

Barton, Jonathan (2006), "Sustentabilidad urbana como planificación estratégica”, Eure, 32 (96), Pontificia Universidad Católica de Chile, Santiago, Chile, pp. 27-45.

Bascuñán, Francisco, Paz Walker y Juan Mastrantonio (2007), "Modelo de cálculo de áreas verdes en planificación urbana desde la densidad habitacional”, Urbano, 10 (15), Universidad del Bio-Bio, Concepción, Chile, pp. 97-101.

Basu, Kaushik (2013), Más allá de la mano invisible, Fondo de Cultura Económica, Ciudad de México, México.

Boudon, Raymond (2004), “Théorie du choix rationnel, théorie de la rationalité limitée ou individualisme méthodologique: que choisir?", Journal des Economistes et des Etudes Humaines, 14 (1), De Gruyter, Berlín, Alemania, pp. 45-62.

Boudon, Raymond (2003), "Beyond rational choice theory", Annual Review of Sociology, vol. 29, Annual Reviews, Massachusetts, Estados Unidos de América, pp. 1-21.

Bougé, Félix (2009), "Caractérisation des espaces verts publics en fonction de leur place dans le gradient urbain-rural”, proyecto de fin de estudios, École Polytechique de l' Université de Tours, Tours, Francia. 
Briseño, Morella, Beatriz Gil, Luz Gómez, Wilver Contreras y Mary Owen (2010), "La ecología urbana y el conocimiento científico", Ecodiseño \& Sostenibilidad, 2 (1), Universidad Politécnica de Valencia-Universidad de Los Andes, España-Venezuela, pp. 373-395, $<$ http://erevistas.saber.ula.ve/index.php/ecodiseno/article/ view/3913/3736>, 13 de febrero de 2017.

Calvo Patrici (2012), "Cooperación y sentimientos morales en el enfoque de racionalidad económica de Elinor Ostrom", XIX Congrés Valencià de Filosofia, 28, 30 y 31 de marzo, Valencia, España.

Camagni, Roberto (2005), Economía urbana, Antoni Bosch, Barcelona, España.

Camagni, Roberto (1999), "El desarrollo urbano sostenible", Papeles de Economía Española, núm. 80, FUNCAS, Madrid, España, pp. 266-290.

Cante, Freddy (2010), Libertades individuales y acción colectiva, Universidad del Rosario, Bogotá, Colombia.

Caponi, Sandra, (2002), "La generación espontánea y la preocupación higienista por la diseminación de los gérmenes", História, Ciências, Saúde-Manguinhos, 9 (3), Fundação Oswaldo Cruz, Río de Janeiro, Brasil, pp. 591-608.

Coleman, James (2011), Fundamentos de teoría social, The College for International Studies, Madrid, España.

Crespo, Ricardo, (2009), "Una reflexión sobre la razón teórica y la razón práctica en la economía”, Revista Empresa y Humanismo, 12 (2), Instituto Empresa y Humanismo, Navarra, España, pp. 107-152.

Dahl, Bernie y Donald Molnar (2003), Anatomy of a park, Waveland Press Inc., Illinois, Estados Unidos de América.

Daly, Herman (2013), "Una economía de estado estacionario", Revista Nueva Sociedad, 1 (144), Foro Nueva Sociedad, Buenos Aires, Argentina, pp. 131-141.

De Mattos, Carlos (2004), "De la planificación a la governance: implicancias para la gestión territorial y urbana", Revista Paranaense de 
Desenvolvimiento, núm. 107, Instituto Paranaense de Desenvolvimento Econômico e Social, Curitiba, Brasil, pp. 9-23.

Echenique, Marcial, Anthony Hargreaves, Gordon Mitchell y Anil Namdeo (2012), "Growing cities sustainably. Does urban form really matter?", Journal of the American Planning Association, 78 (2), American Planning Association, Chicago, Estados Unidos de América, pp. 121-137.

Elster, Jon (2003), Tuercas y tornillos, Gedisa, Ciudad de México, México.

Engels, Friedrich (2010), The condition of the working-class in England in 1844, Cambridge, Londres, Inglaterra.

Falcón, Antoni (2007), Espacios verdes para una ciudad sostenible, Gustavo Gili, Barcelona, España.

Fan, Yingling, Kirti Das y Qian Chen (2011), "Neighborhood green, social support, physical activity, and stress: assessing the cumulative impact", Health \& Place, 17 (6), University of Edinburgh, Edinburgh, Reino Unido, pp. 1202-1211.

Fernández, José (2006), Planificación estratégica de ciudades, Reverté, Barcelona, España.

Fernández, Federico (2000), Europa y el urbanismo neoclásico en la Ciudad de México, Plaza y Valdés, Ciudad de México, México.

Field, Barry y Martha Field (2016), Environmental Economics, McGrawHill, New York, Estados Unidos de América.

Freeman, Myrick, Joseph Herriges y Catherine Kling (2014), The measurement of environmental and resource values, RFF Press, New York, Estados Unidos de América.

Frutos de, Pablo y Sonia Esteban (2009), "Estimación de los beneficios generados por los parques y jardines urbanos a través del método de valoración contingente", Revista de Economía Pública Urbana, 1 (10), Universidad Santiago de Compostela, Santiago de Compostela, España, pp. 13-51. 
Fujita, Masahisa, Paul Krugman y Anthony Vanables (2001), The spatial economy, MIT Press, Massachusetts, Estados Unidos de América.

Fusco, Luigi, Maria Cerreta, Pasquale de Toro y Fabiana Forte (2009), "The human sustainable city: values, approaches and evaluative tools", en Mark Deakin, Gordon Mitchell, Peter Nijkamp y Ron Vreeker (eds.), Sustainable Urban Development. Volume 2: The environmental assessments methods, Routledge, Cornwall, Gran Bretańa, pp. 65-93.

Gámez, Vicente (2005), “Sobre sistemas, tipologías y estándares de áreas verdes en el planeamiento urbano", Revista de Diseño Urbano y Paisaje, (2) 6, Universidad Central de Chile, Santiago, Chile, pp. 1-22, <http://www.ucentral.cl/du\&p/pdf/000002.pdf>, 13 de febrero de 2017.

Garvin, Alexander (2013), The american city: what works, what doesn't, McGraw-Hill Professional, New York, Estados Unidos de América.

Garvin, Alexander (2010), Public parks: the key to livable communities, W. W. Norton \& Company, New York, Estados Unidos de América.

Gehl Jan y Birgitte Svarre (2013), How to study public life, Island Press, Washington, Estados Unidos de América.

González, Coral (2011), "Una introducción a la racionalidad limitada y la economía del comportamiento", Gaceta de Economía, núm. 28, Instituto Tecnológico Autónomo de México, Ciudad de México, México, pp. 321-328.

Gordillo, Gustavo (2014), "Estado, mercado, comunidad: Ostrom, la controversial”, Revista Mexicana de Ciencias Sociales, 76 (5), Universidad Nacional Autónoma de México, Ciudad de México, México, pp. 227-257.

Gutiérrez, Juan (2013), "La planeación urbana en México: retos y perspectivas desde la agenda internacional", Revista de urbanismo, 1 (29), Universidad de Chile, Santiago, Chile, pp. 2-16.

Gutiérrez, Juan (2009), "Planeación urbana en México: un análisis crítico sobre su proceso de evolución”, Urbano, 12 (19), Universidad del Bio-Bio, Concepción, Chile, pp. 52-63. 
Gutiérrez, Esthela y Édgar González (2010), De las teorías del desarrollo al desarrollo sustentable, Siglo XXI, Monterrey, México.

Hardy, Dennis (2005), "Garden cities: practical concept, elusive reality", Journal of Planning History, 4 (4), Sage, California, Estados Unidos de América, pp. 383-391.

Harnik, Peter (2010), Urban green: innovative parks for resurgent cities, Island Press, Washington, Estados Unidos de América.

Hernández, Tania (2008), "Breve exposición de las contribuciones de Georgescu Roegen a la economía ecológica y un comentario crítico", Argumentos, 21 (56), Universidad Autónoma Metropolitana, Ciudad de México, México, pp. 35-52.

Instituto Nacional de Cultura (2007), Documentos fundamentales para el patrimonio cultural, Instituto Nacional de Cultura, Lima, Perú.

Jim, C. Y. y Wendy Chen, (2006), "Recreation-amenity use and contingent valuation of urban greenspaces in Guangzhou, China", Landscape and Urban Planning, 75 (1-2), Elsevier, Amsterdam, Países Bajos, pp. 81-96.

Jirón, Paola y Pablo Mansilla (2014), "Las consecuencias del urbanismo fragmentador en la vida cotidiana de habitantes de la ciudad de Santiago de Chile", Eure, 40 (121), Pontificia Universidad Católica de Chile, Santiago, Chile, pp. 5-28.

Kallis, Giorgios y Richard Norgaard (2010), "Coevolutionary ecological economics”, Ecological Economics, 69 (4), Elsevier, Amsterdam, Países Bajos, pp. 690-699.

Kaźmierczak, Aleksandra (2013), "The contribution of local parks to neighbourhood social ties", Landscape and Urban Planning, 109 (1), Elsevier, Amsterdam, Países Bajos, pp. 31-44.

Konijnendijk, Cecil, Matilda Annerstedt, Anders Nielsen y Sreetheran Maruthaveeran (2013), Benefits of urban parks: a systematic review. A report for IFPRA, IFPRA, Copenhague, Dinamarca.

Layuno, Ángeles (2013), "Las primeras ciudades de la industria: trazados urbanos, efectos territoriales y dimensión patrimonial. La 
experiencia de Nuevo Baztán (Madrid)", Scripta Nova Revista Electrónica de Geografía y Ciencias Sociales, 17 (451), Universidad de Barcelona, Barcelona, España, <http://www.ub.edu/geocrit/ sn/sn-451.htm> 13 de febrero de 2017.

Lee, Andrew y Ravi Maheswaran (2010), "The health benefits of urban green spaces: a review of the evidence", Journal of Public Health, 33 (2), Oxford UP, Oxford, Reino Unido, pp. 212-222.

Lehtonen, Markku (2004), "The environmental-social interface of sustainable development: capabilities, social capital, institutions", Ecological Economics, 49 (2) Elsevier, Amsterdam, Países Bajos, pp. 199-214.

Lozano, Rodrigo (2008), "Envisioning sustainability three-dimensionally", Journal of Cleaner Production, 16 (8), University of Tennessee, Tennessee, Estados Unidos de América, pp. 1838-1846.

Martínez, Joan y Jordi Roca (2001), Economía ecológica y politica ambiental, Fondo de Cultura Económica, Ciudad de México, México.

Medina, María (2010), "La práctica del paisajismo", Estudios Geográficos, 71 (269), Consejo Superior de Investigaciones Científicas, Madrid, España, pp. 633-658, <http://www.ub.edu/geocrit/sn/sn451.htm>, 13 de febrero de 2017.

McLoughling, Brian (1969), Urban \& Regional Planning, Faber \& Faber, Londres, Inglaterra.

Meadows, Donella, Jorgen Randers y Dennis Meadows (2006), Los limites del crecimiento 30 años después, Galaxia Gutenberg, Barcelona, España.

Mebratu, Desta (1998), "Sustainability and sustainable development: historical and conceptual review", Environmental Impact Assesment Review, núm. 18, University of East Anglia, Norwich, Inglaterra, pp. 493-520.

Mumford, Lewis (2014a), La ciudad en la historia, Pepitas de Calabaza, Logroño, España. 
Mumford, Lewis (2014b), La carretera y la ciudad, Godot, Buenos Aires, Argentina.

North, Douglass (2006), Instituciones, cambio institucional y desempeño económico, Fondo de Cultura Económica, Ciudad de México, México.

North, Douglass (1993), "What do we mean by rationality?", Public Choice, 77 (1), Springer, Berlín, Alemania, pp. 159-162.

Nunes, Paulo and Peter Nijkamp (2011), "Economic valuation, values and contingent method: an overview”, Regional Science Inquiry Journal, 3 (1), HARS, Atenas, Grecia, pp. 95-116.

Olmsted, Frederick (2015), Frederick Law Olmsted, landscape architect, 1822-1903, Hard Press, Miami, Estados Unidos de América.

ONU (Organización de las Naciones Unidas) (2015), "Habitat III Issue Papers, 11 - Public Space, ONU”, UN Task Team writeshop held in New York, 26-29 de mayo, New York, Estados Unidos de América.

Ostrom, Elinor (2015), Comprender la diversidad institucional, Fondo de Cultura Económica, Ciudad de México, México.

Ostrom, Elinor (2010), "Beyond markets and states: Polycentric governance of complex economic systems", American Economic Reviews, 100 (3), American Economic Association, Pittsburgh, Estados Unidos de América, pp.1-33.

Paskaleva-Shapira, Krassimira (2009), "Governing the sustainability of urban development", en Ron Vreeker, Mark Deakin y Stephen Curwell (eds.), Sustainable Urban Development. Volumen 3: The Toolkit for Assessment, Routledge, Cornwall, Gran Bretańa, pp. 248-273.

Pedrajas, Marta (2006), "La transformación ética de la racionalidad económica en Amartya Sen. Una recuperación de Adam Smith", Quaderns de filosofia i ciència, núm. 36, Universidad de Valencia, Valencia, España, pp. 105-117. 
Pierri, Naina (2005), "Historia del concepto de desarrollo sustentable", en Guillermo Foladori y Naina Pierri, (coords.), ¿Sustentabilidad? Desacuerdos sobre el desarrollo sustentable, Miguel Ángel Porrúa, Ciudad de México, México, pp. 27-81.

Ponting, Clive (1992), Historia verde del mundo, Paidós, Barcelona, España.

Reyes, Sonia e Isabel Figueroa (2010), "Distribución, superficie y accesibilidad de las áreas verdes en Santiago de Chile", Eure, 36 (109), Pontificia Universidad Católica de Chile, Santiago, Chile, pp. 89-110.

Rocuts, Asthriesslav, Luis Jiménez y Marcela Navarrete (2009), "Interpretaciones visuales de la sostenibilidad: enfoques comparados y presentación de un modelo integral para la toma de decisiones", Revista Internacional de Sostenibilidad, Tecnología y Humanismo, 1 (4), Universitat Politècnica de Catalunya, Barcelona, España, pp. 1-22, <http://upcommons.upc.edu/bitstream/handle/2099/8527/rocuts.pdf?sequence=1\&isAllowed $=y>$, 13 de febrero de 2017.

Rodríguez, Juan (2011), "Planificación urbana en perspectiva: una mirada a nuestra formación en teoría de la planificación urbana", Quivera, 13 (2), Universidad Autónoma del Estado de México, Toluca, México, pp. 232-258.

Salas, Camilo (2013), "La noción de libertad en Amartya Sen y la economía política latinoamericana", Revista Panameña de Política, núm. 16, CID, Panamá, Panamá, pp. 19-40.

Salvador Pedro (2003), La planificación verde en ciudades, Gustavo Gili, Barcelona, España.

San Emeterio, Nieves (2014), Nueva economía institucional, Síntesis, Madrid, España.

Sen, Amartya (1977), "Rational fools: a critique of the behavioral foundations of economic theory", Philosophy \& Public Affairs, 6 (4), Princeton University Press, New York, Estados Unidos de América, pp. 317-344. 
Simon, Herbert (1985), "Human nature in politics: the dialogue of psychology with political science", The American Political Science Review, 79 (2), Cambridge, Londres, Inglaterra, pp. 293-304.

Singh Vijai Shanker, Deep Narayan Pandey y Pradeep Chaudhry (2010), Urban forests and open green spaces: lessons for Jaipur, Rajasthan, India, Rajasthan State Pollution Control Board, Jaipur, India.

Sorensen, Mark, Valerie Barzetti, Kari Keipi y John Williams (1998), Manejo de las áreas verdes urbanas, documento de buenas prácticas 109, BID, Washington, Estados Unidos de América.

Steuer, Max (2000), "A hundred years of town planning and the influence of Ebenezer Howard”, British Journal of Sociology, 51 (2), Wiley, New York, Estados Unidos de América, pp. 377-386.

Venkatachalam, L. (2007), "Environmental economics and ecological economics: where they can converge?", Ecological Economics, 61 (2) Elsevier, Amsterdam, Países Bajos, pp. 550-558.

Venkatachalam, L. (2004), "The contingent valuation method: a review”, Environmental Impact Assessment Review, 24 (1) Elsevier, Amsterdam, Países Bajos, pp. 89-124.

Vojnovic, Igor (2014), "Urban sustainability: research, politics, policy and practice", Cities, 41 (1), Elsevier, Amsterdam, Países Bajos, pp. 30-44.

Vreeker, Ron (2009), "Sustainable urban development. The case of mixed and compact land use", en Ron Vreeker, Mark Deakin y Stephen Curwell (eds.), Sustainable Urban Development. Volume 3: The toolkit for assessment, Routledge, Cornwall, Gran Bretaña, pp. 85-102.

Wackernagel, Mathis y William Rees (2001), Nuestra huella ecológica, Lom, Santiago, Chile.

Wall, Derek, (2014), The Sustainable Economics of Elinor Ostrom: commons, contestation and craft, Routledge, New York, Estados Unidos de América. 
Wang Xiao-Jun (2009), "Analysis of problems in urban green space system planning in China", Journal of Forestry Research, 20 (1), Springer, Berlín, Alemania, pp. 79-82.

WCED (The World Commission on Environment and Development) (2009), Our common future, Oxford, Oxford, Inglaterra.

Weber, Max (2014), Economía y Sociedad, Fondo de Cultura Económica, Ciudad de México, México.

Webster, Premila y Denise Sanderson (2012), "Healthy cities indicators a suitable instrument to measure health?", Journal of Urban Health: Bulletin of the New York Academy of Medicine, 90 (1), Academy of Medicine, New York, Estados Unidos de América, pp. 52-61.

Whitehead, John y Glenn Blomquist (2009), "The use of contingent valuation in benefit-cost analysis", en Anna Alberini y James Kahn, (eds.), Handbook on Contingent Valuation, EE Publishing, Cheltenham, Reino Unido, pp. 92-115.

WHO (2010), Urban planning, environment and health, WHO Regional Office for Europe, Copenhague, Dinamarca.

Williamson, Oliver (2013), Las instituciones económicas del capitalismo, Fondo de Cultura Económica, Ciudad de México, México.

Wilson, William (1994), The city beautiful movement, Johns Hopkins UP, Baltimore, Estados Unidos de América.

Wolch, Jennifer, Jason Byrne y Joshua Newell (2014), “Urban green space, public health, and environmental justice: the challenge of making cities just green enough", Landscape and Urban Planning, (125), Elsevier, Amsterdam, Países Bajos, pp. 234-244.

Young, Robert (2012), "Planting the living city", Journal of the American Planning Association, 77 (4), American Planning Association, Chicago, Estados Unidos de América, pp. 368-381.

Young, Pablo, Verónica Hortis, Maria Chambi y Bárbara Finn, (2011), "Florence Nightingale (1820-1910), a 101 años de su fallecimiento", Revista Médica de Chile, 139 (6), Sociedad Médica de Santiago, Santiago, Chile, pp. 807-813. 
Zhang XiuJuan y LiHua Zhou (2012), "Development of contingent valuation method in evaluating non-market values of resources and environment in China", Sciences in Cold and Arid Regions, 4 (6), Chinese Academy of Sciences, Lanzhou, China, pp. 536-543.

Recibido: 10 de julio de 2015. Corregido: 10 de enero de 2016. Aceptado: 31 de enero de 2017.

Ramiro Flores-Xolocotzi. Es doctor en Ciencias por el Colegio de Postgraduados, actualmente es docente en la Universidad La Salle, Ciudad de México, Facultad de Ciencias Químicas. Entre otras actividades es consultor en temas de economía y manejo de recursos naturales. Sus líneas de investigación actual son: Gestión y planificación de espacios verdes urbanos y Gestión de servicios públicos y recursos de uso común: agua potable y mantos acuíferos. Sus tres más recientes publicaciones son: "El valor económico del uso recreativo que presta el Parque Ambiental Bicentenario en Metepec, Estado de México (México)", Economía y Sociedad 1 (30), Universidad Michoacana de San Nicolás de Hidalgo, Morelia, México, pp. 15-32 (2014); "Incorporando desarrollo sustentable y gobernanza en la gestión y planificación de áreas verdes urbanas”, Frontera Norte, 24 (48), Colegio de la Frontera Norte, Tijuana, México, pp. 165190 (2012) y "Análisis de los gustos recreativos en el Parque Ambiental Bicentenario de Metepec, Estado de México (México)", PASOS Revista de Turismo y Patrimonio Cultural, 10 (3), Universidad de La Laguna, Islas Canarias, Espańa, pp. 315-329 (2012). 